\title{
Fat Necrosis
}

National Cancer Institute

\section{Source}

National Cancer Institute. Fat Necrosis. NCI Thesaurus. Code C26772.

Destruction of adipose tissue caused by the activity of lipase on damaged fat cells. 